\title{
Inhibition of neddylation by MLN4924 improves neointimal hyperplasia and promotes apoptosis of vascular smooth muscle cells through p53 and p62
}

\author{
Tang-Jun Ai ${ }^{1,2,3,6}$, Jian-Yong Sun ${ }^{1,2,6}$, Lin-Juan Du ${ }^{1,2,3,6}$, Chaoji Shi ${ }^{1,2}$, Chao Li ${ }^{1,2,3}$, Xue-Nan Sun ${ }^{1,2,3}$, Yan Liu ${ }^{1,2}$, Lihui Li ${ }^{4}$, Zhixiong Xia ${ }^{5}$, \\ Lijun Jia ${ }^{4}$, Jianmiao Liü ${ }^{\star, 5}$ and Sheng-Zhong Duan ${ }^{\star, 1,2}$
}

Targeting apoptosis of vascular smooth muscle cells (VSMCs) represents an attractive approach to diminish the occurrence of restenosis. Neddylation is a highly conserved post-translational modification process and inhibition of neddylation has been shown to regulate apoptosis of other cells. However, the impacts of neddylation inhibition on VSMCs and neointimal hyperplasia have not been studied. In our present study, we have shown that MLN4924, a selective inhibitor of NEDD8-activating enzyme (NAE), markedly inhibited neointimal hyperplasia and accumulation of VSMCs, whereas increased apoptosis in the vascular wall. In vitro studies revealed that MLN4924 induced G2/M arrest and apoptosis of human VSMCs. Knockdown of NAE1 had similar effects. MLN4924 upregulated p53 and p62 in human VSMCs. Knockdown of either p53 or p62 mitigated the impacts of MLN4924 on G2/M arrest and apoptosis. Moreover, p53 knockdown abolished MLN4924-induced upregulation of p62. Finally, smooth muscle p53 knockout mice were generated and subjected to femoral artery injury and MLN4924 treatment. Deficiency of p53 in smooth muscle blocked the effects of MLN4924 on neointimal hyperplasia and apoptosis. Together, our results revealed that neddylation inhibition induces apoptosis through p53 and p62 in VSMCs and improves neointimal hyperplasia mainly by promoting apoptosis through smooth muscle p53 in mice. These pre-clinical data provide strong translational implications for targeting restenosis by perturbation of neddylation using MLN4924.

Cell Death and Differentiation (2018) 25, 319-329; doi:10.1038/cdd.2017.160; published online 13 October 2017

Percutaneous coronary intervention $(\mathrm{PCl})$ is widely used to treat coronary heart disease. ${ }^{1,2}$ However, the procedure itself causes vascular injury that may lead to restenosis, a major limitation of this therapeutic approach. ${ }^{3}$ Proliferation and migration of vascular smooth muscle cells (VSMCs) have been considered two of the most important characteristics in the process of restenosis. ${ }^{4,5}$ However, even though the use of paclitaxel and rapamycin, both of which inhibit the proliferation and migration of VSMCs and significantly lowers the incidence of restenosis, ${ }^{6,7}$ the rate of restenosis remains above $12 \%$. ${ }^{8,9}$ Therefore, new strategies are still in need to achieve better outcomes.

Targeting VSMC apoptosis may be a feasible strategy to further diminish the occurrence of restenosis. It has been recognized that besides proliferation and migration, apoptosis of VSMCs also has important roles in restenosis. ${ }^{10}$ For example, an early study has shown that overexpression of fas ligand induces apoptosis of VSMCs and inhibits neointimal hyperplasia in balloon-injured rat carotid arteries. ${ }^{11}$ Later efforts have demonstrated that agents such as statins promote apoptosis of VSMCs and inhibit neointimal hyperplasia in animal models and are generally considered beneficial for restenosis in human patients after $\mathrm{PCl}$, although clinical data are not always consistent. ${ }^{12}$ Recent data have revealed that VSMCs are resistant to apoptosis during the development of neointimal hyperplasia and that alleviation of such resistance is highly effective in facilitation of VSMC apoptosis and reduction of neointima formation. ${ }^{13}$ These studies have suggested that induction of VSMC apoptosis is of therapeutic interests for treating restenosis.

Neddylation, a post-translational protein modification that adds neural precursor cell expressed developmentally downregulated protein 8 (Nedd8) to target proteins, has been demonstrated to exert critical functions in cell proliferation, growth, senescence, autophagy, and apoptosis. ${ }^{14,15}$ Similar to ubiquitin, Nedd8 is conjugated to its substrates through an enzymatic cascade that consists of an E1-activating enzyme, an E2 conjugating enzyme, and an E3 ligase. ${ }^{16}$ Substrates of

\footnotetext{
'Laboratory of Oral Microbiota and Systemic Diseases, Ninth People's Hospital, School of Stomatology, Shanghai Jiao Tong University School of Medicine, Shanghai 200011, China; ${ }^{2}$ Shanghai Key Laboratory of Stomatology \& Shanghai Research Institute of Stomatology; National Clinical Research Center of Stomatology, Shanghai Jiao Tong University School of Medicine, Shanghai 200011, China; ${ }^{3}$ Key Laboratory of Nutrition and Metabolism, Institute for Nutritional Sciences, Shanghai Institutes for Biological Sciences, Chinese Academy of Sciences, University of the Chinese Academy of Sciences, Shanghai 200031, China; ${ }^{4}$ Cancer Institute, Longhua Hospital, Shanghai University of Traditional Chinese Medicine, Shanghai 200032, China and ${ }^{5}$ Cellular Signaling Laboratory, Key Laboratory of Molecular Biophysics of Ministry of Education, Huazhong University of Science and Technology, Wuhan 430074, China

*Corresponding author: J Liu, College of Life Science \& Technology, Huazhong University of Science and Technology, 1037 Luoyu Road, Wuhan 430074, China. Tel: +86 27 87792024; Fax: +86 27 87792118; E-mail: jmliu@ @ust.edu.cn

or S-Z Duan, Laboratory of Oral Microbiota and Systemic Diseases, Ninth People's Hospital, School of Stomatology, Shanghai Jiao Tong University School of Medicine, 639 Zhizaoju Road, Shanghai 200011, China. Tel: +86 2123271699 Ext 5962; Fax: +86 21 63136856; E-mail: duansz@shsmu.edu.cn

${ }^{6}$ These authors contributed equally to this work.

Received 29.11.16; revised 27.8.17; accepted 29.8.17; Edited by Y Shi; published online 13.10.17
} 
a
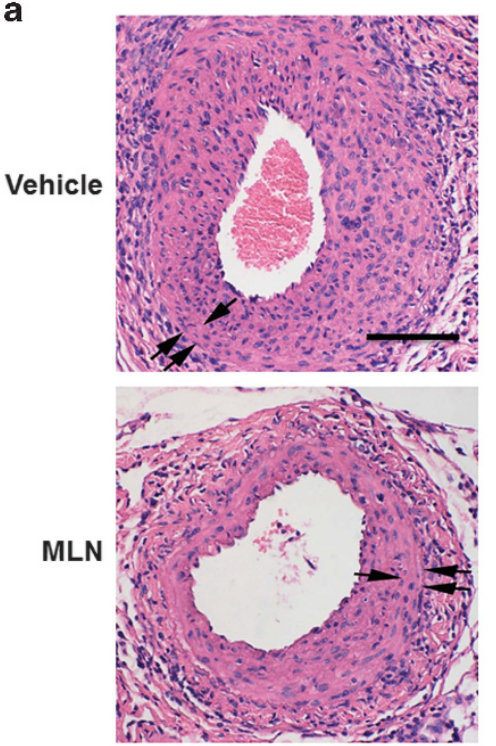

C

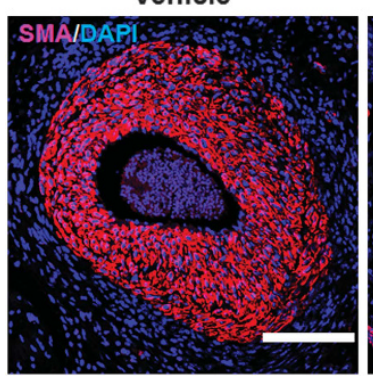

b
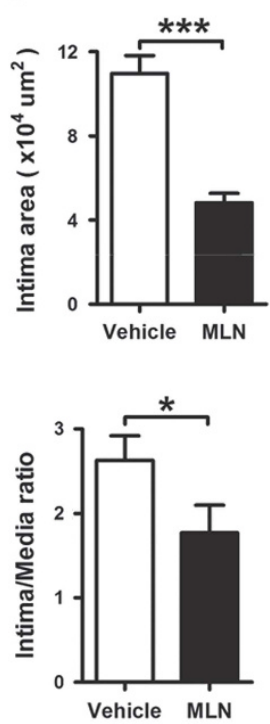
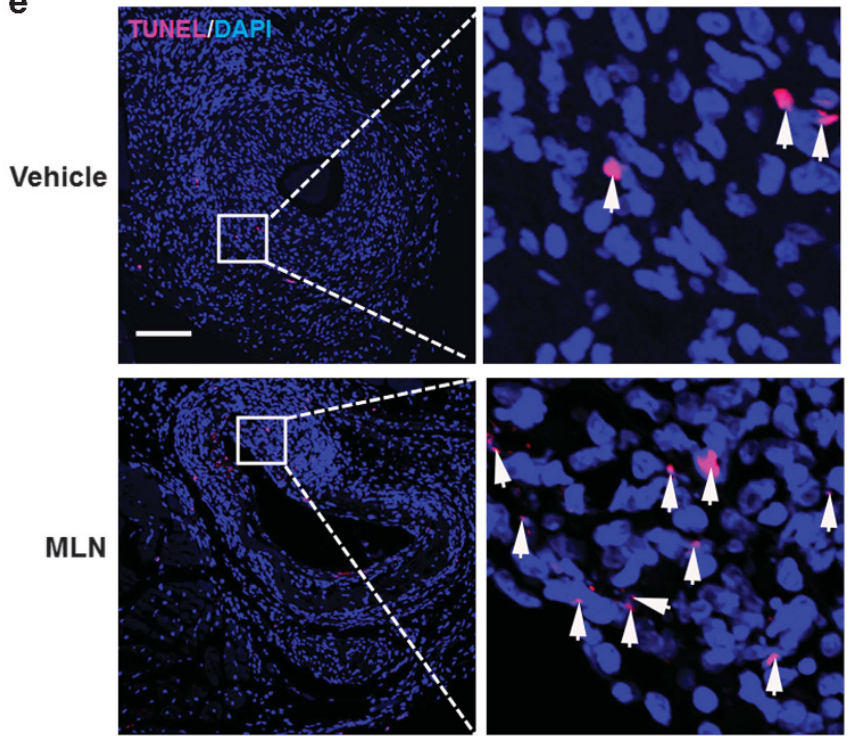

d

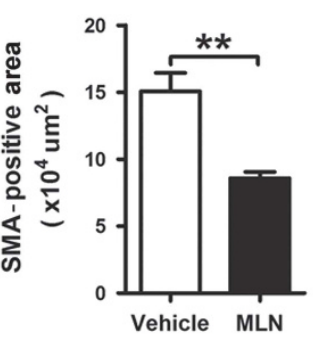

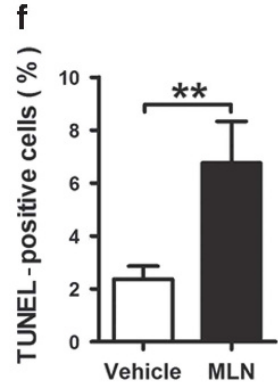

Figure 1 MLN4924 inhibits arterial injury-induced neointimal hyperplasia in mice. (a) Representative hematoxylin and eosin staining of cross-sections of femoral arteries from wire-injured C57 mice that were treated with vehicle or MLN4924 (MLN) for 28 days. Single arrows indicate internal elastic lamina and double arrows indicate external elastic lamina. (b) Quantifications of intimal area and intima/media ratio. (c) Representative immunofluorescence staining of $\alpha$-smooth muscle actin (SMA) in injured arteries. (d) Quantification of SMA-positive cells. (e) Representative TUNEL staining of injured arteries. Arrows denote TUNEL-positive cells. (f) Quantification of TUNEL index as percentage of apoptotic cells. All scale bars: $280 \mu \mathrm{m} . n=6 .{ }^{*} P<0.05,{ }^{\star \star} P<0.01,{ }^{\star \star \star} P<0.001$

neddylation include cullin family proteins and transcription factors such as p53, p73, and E2F1. ${ }^{14,17}$ Studies have shown that MLN4924, an inhibitor of Nedd8-activating enzyme (NAE), ${ }^{18,19}$ strikingly suppresses the growth of tumor cells through inducing senescence, autophagy, and/or apoptosis. $^{20-22}$ We therefore hypothesize that interfering neddylation may improve neointimal hyperplasia by inhibiting the growth of VSMCs.

To test the hypothesis, we first used a classical femoral wire injury mouse model that simulated the pathological changes after PCI to investigate whether MLN4924 improved neointimal hyperplasia and its effects on apoptosis of VSMCs. Then, we studied the cellular mechanisms by evaluating the impacts of MLN4924 and knockdown of NAE subunit 1 (NAE1) on cell cycle and apoptosis of human smooth muscle cells (hSMCs). Finally, we further explored the intrinsic molecular mechanisms that mediated the effects of MLN4924 in vitro and in vivo.

\section{Results}

MLN4924 inhibits arterial injury-induced neointimal hyperplasia in mice. In a wire injury mouse model,
MLN4924 significantly decreased intima area and intima/ media ratio of injured femoral arteries compared with vehicle control (HPBCD) (Figures 1a and b). Further, smooth muscle actin (SMA)-positive cells were reduced in injured femoral arteries of MLN4924-treated mice (Figures 1c and d), indicating less accumulation of VSMCs. Apoptosis is a major factor that determines the amount of VSMCs in the neointima after vascular injury. TdT-mediated dUTP nick end labeling (TUNEL)-positive cells were significantly increased in the arteries of MLN4924-treated mice (Figures $1 \mathrm{e}$ and $\mathrm{f}$ ), whereas SMA and Ki67 double-positive cells did not differ between the two groups (Supplementary Figure S1), suggesting that MLN4924 mainly increased apoptosis of VSMCs. The two groups showed comparable body weights (Supplementary Figure S2). These results suggested that MLN4924 inhibited neointimal hyperplasia by promoting apoptosis of VSMCs.

MLN4924 and NAE1 knockdown both induce G2/M arrest and apoptosis in hSMCs. We then studied the impacts of MLN4924 on the growth of hSMCs to better understand the cellular and molecular mechanisms. Fluorescence-activated 
a

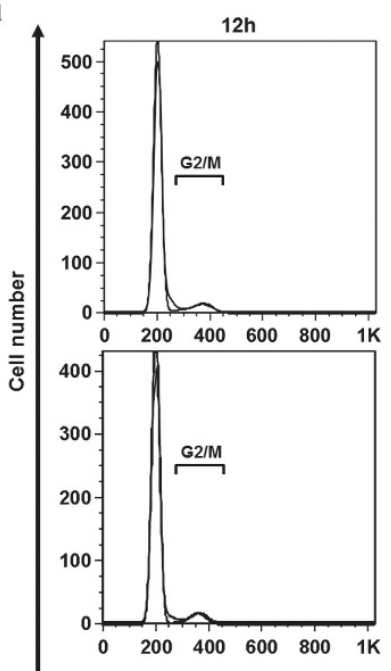

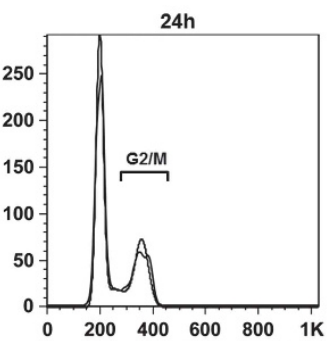

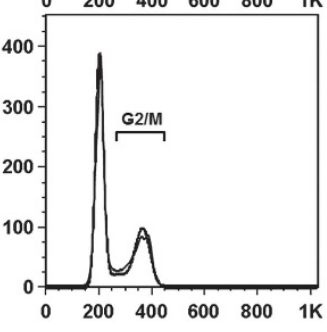

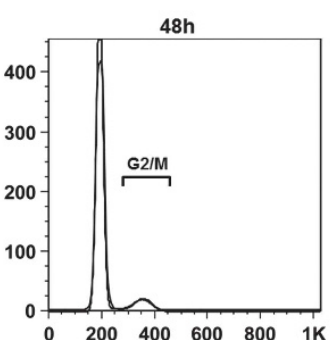

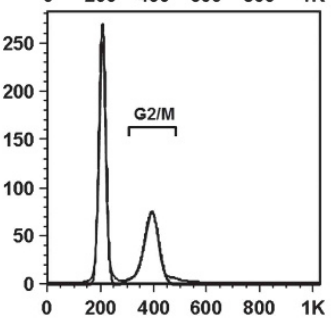

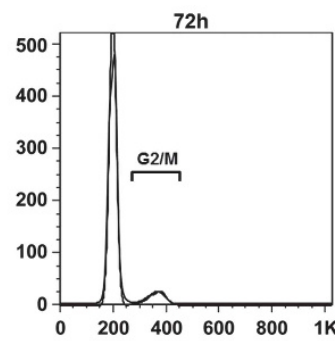

DMSO

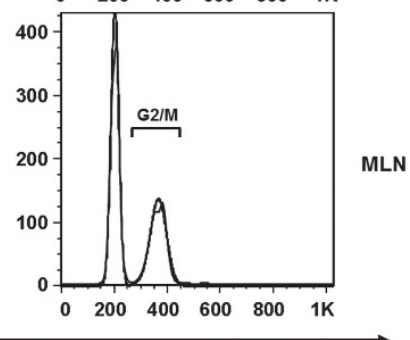

Propidium lodide
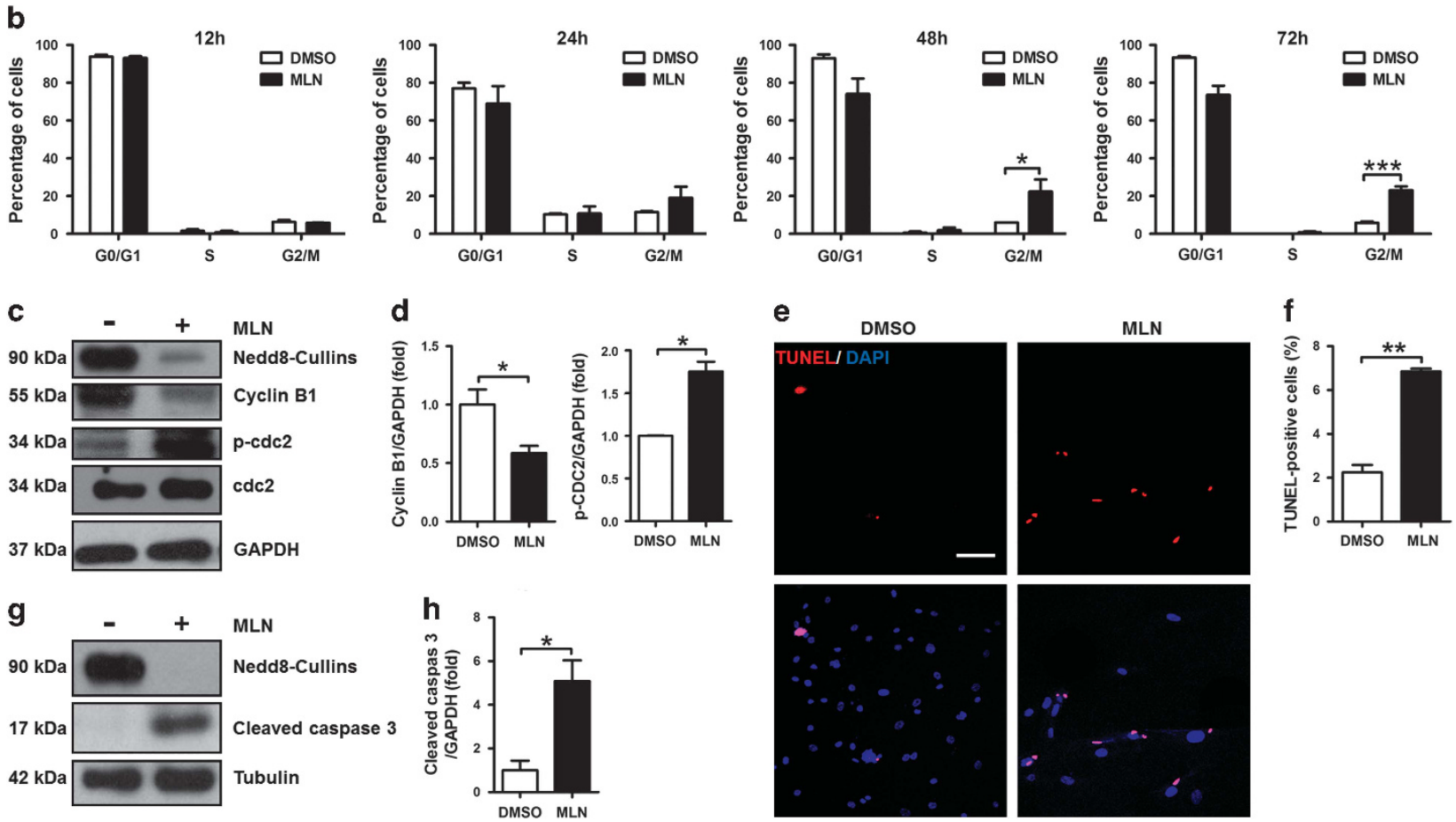

Figure 2 MLN4924 induces G2/M arrest and apoptosis in human smooth muscle cells (hSMCs). (a) FACS analysis of cell cycle of hSMCs treated with vehicle (DMSO) or MLN4924 (MLN). (b) Quantification of percentages of hSMCs in different cell cycle phases at different time points. (c) Western blotting analysis of Nedd8-cullins, cyclin B1, p-cdc2, and total cdc2 in hSMCs. GAPDH was used as a loading control. (d) Quantification of (c). (e) TUNEL staining of hSMCs. (f) Quantification of TUNEL-positive cells. (g) Western blotting analysis of Nedd8-cullins and cleaved caspase 3 in hSMCs. (h) Quantification of (g). For (c-h), cells were treated for $72 \mathrm{~h}$. Scale bar: $300 \mu \mathrm{m}$. Each experiment was repeated at least three times. ${ }^{*} P<0.05,{ }^{* \star} P<0.01,{ }^{* * *} P<0.001$

cell sorting (FACS) analysis showed that hSMCs in G2/M phase peaked at $24 \mathrm{~h}$ after DMSO treatment (Figures 2a and b), likely reflecting the peak of proliferation and consistent with previous observations. ${ }^{23}$ The hSMCs in G2/M phase were markedly increased by MLN4924 treatment, particularly at $48 \mathrm{~h}$ and $72 \mathrm{~h}$ time points (Figures $2 \mathrm{a}$ and $\mathrm{b}$ ). Cyclin B1cdc2 is a pair of key regulators that promote G2/M phase and phosphorylation of cdc2 leads to its inhibition. ${ }^{24}$ Consistently, the protein level of cyclin B1 was decreased and that of phosphorylated cdc2 (p-cdc2) was increased by MLN4924 treatment (Figures $2 \mathrm{c}$ and $\mathrm{d}$ ). These data demonstrated that MLN4924-induced G2/M arrest in hSMCs.

TUNEL-positive cells were significantly increased by MLN4924 in hSMCs (Figures 2e and f). Expression of cleaved caspase 3 was also upregulated by MLN4924 (Figures $2 \mathrm{~g}$ and $h$ ), further suggesting that MLN4924-induced apoptosis in hSMCs. The effectiveness of MLN4924 was confirmed by the profoundly suppressed protein level of Nedd8-conjugated 
proteins and specifically Nedd8-conjugated cullins (Figures 2c and g; Supplementary Figure S3). Taken together, MLN4924 inhibited the growth of hSMCs by inducing G2/M arrest and apoptosis.

We further used small interfering RNA (siRNA)-mediated knockdown of NAE1 to determine the impacts of neddylation inhibition on hSMCs. The efficiency of NAE1 knockdown was verified by QRT-PCR (Figure 3a). Furthermore, NAE1 knockdown led to a drastic decrease of Nedd8-Cullins, the neddylated form of Cullin proteins (Figure $3 b$ ), suggesting the inactivation of neddylation. Similar to the effects of MLN4924, transfection of NAE1 siRNA in hSMCs caused robust increase of cells in G2/M phase (Figures $3 c$ and d), TUNEL-positive cells (Figures $3 e$ and $f$ ), and expression of cleaved caspase 3 (Figures $3 g$ and $h$ ).

p53 mediates the effects of MLN4924 on G2/M arrest and apoptosis in VSMCs. MLN4924 induces apoptosis of tumor cells through DNA re-replication and damage ${ }^{25}$ We therefore tested whether DNA damage and checkpoint activation occurred in hSMCs treated with MLN4924. Indeed, MLN4924 increased phosphorylation of H2AX, a marker of DNA damage, in hSMCs (Figures $4 a$ and b). Consequently, MLN4924 markedly elevated the phosphorylation and total level of p53, a downstream target of checkpoint activation (Figures $4 a$ and $b$ ), although it did not cause obvious change at mRNA level (Figure 4c). Knockdown of NAE1 by siRNA also induced upregulation of $\mathrm{p}-\mathrm{H} 2 \mathrm{AX}$ and $\mathrm{p} 53$ (Supplementary Figure S4), suggesting that MLN4924induced DNA damage was at least in part because of a reduction in neddylation. Furthermore, treatment of MLN4924 notably increased protein expression of chromatin licensing and DNA replication factor 1 (Cdt1) in hSMCs (Supplementary Figure S5). Cdt1 is a known CRL substrate and its overexpression has been shown to induce DNA damage response. ${ }^{26,27}$ Interestingly, knockdown of Cdt1 inhibited MLN4924-induced upregulation of p-H2AX, p53, and cleaved caspase 3 in hSMCs (Supplementary Figure S6), indicating that Cdt1 at least partially mediated the impacts of MLN4924 on DNA damage response, p53 induction, and apoptosis of these cells.

Previous studies have demonstrated that p53 has crucial roles in regulating $\mathrm{G} 2 / \mathrm{M}$ arrest and apoptosis. ${ }^{28} \mathrm{We}$ then explored whether p53 mediated these effects of MLN4924 in hSMCs. siRNA-mediated knockdown of p53 (Figures 4c and d) markedly attenuated MLN4924-induced increase of hSMCs in $\mathrm{G} 2 / \mathrm{M}$ phase (Figures $4 \mathrm{e}$ and $\mathrm{f}$ ), supporting that p53 mediated the effects of MLN4924 on G2/M arrest. TUNELpositive cells were also significantly decreased by p53 knockdown in MLN4924-treated hSMCs (Figures 4g and h), suggesting that p53 mediated the effects of MLN4924 on apoptosis. Moreover, the upregulation of p53 target genes such as fas and p21, both of which were closely related to apoptosis and growth arrest, was blocked by p53 siRNA (Figure 4i). Under the treatment of MLN4924, the protein level of cleaved caspase 3 was drastically lower in primary VSMCs from p53 KO mice compared with those from control mice (Supplementary Figure S7), further suggesting that p53 mediated the effects of MLN4924 on apoptosis of VSMCs.
These results together demonstrated that MLN4924 induced G2/M arrest and apoptosis through p53 in VSMCs.

p62 mediates the effects of MLN4924 on apoptosis in VSMCs. MLN4924 induces autophagy in multiple cancer cell lines. $^{20,22,29}$ During autophagy, microtubule-associated protein 1 light chain 3-I (LC3-I) is converted to lipidated LC3-II. ${ }^{30}$ Western blotting analysis showed that the conversion of LC3I to LC3-II was not increased, suggesting that MLN4924 did not induce autophagy in hSMCs (Supplementary Figure S8). Accumulation of p62 (or sequestosome 1, SQSTM1) is an indicator of suppressed autophagy. ${ }^{30}$ Both mRNA and protein levels of p62 were upregulated by MLN4924 treatment, further indicating that MLN4924 did not increase autophagy in hSMCs (Figures 5a-c). Studies have shown that p62 also mediates the activation of apoptosis. ${ }^{31,32}$ siRNA-mediated knockdown of p62 (Figure 5d) significantly decreased the number of TUNEL-positive cells in MLN4924-treated hSMCs (Figures 5e and f). Consistently, expression of cleaved caspase 3 was markedly decreased by p62 knockdown, further implying that p62 mediated MLN4924-induced apoptosis of hSMCs (Figures $5 \mathrm{~g}$ and $\mathrm{h}$ ). However, knockdown of p62 did not affect MLN4924-induced G2/M arrest of hSMCs (Supplementary Figure S9). At baseline without MLN4924 treatment, p62 knockdown did not affect apoptosis of hSMCs (Supplementary Figure S10). These data indicated that p62 mediated the pro-apoptotic effects of MLN4924 without affecting G2/M arrest in hSMCs.

Notably, p53 knockdown by siRNA blocked MLN4924induced upregulation of p62 (Figures 5i-k), suggesting that p53 mediated the impacts of MLN4924 on p62 in regulating apoptosis of hSMCs. Moreover, knockdown of p62 in addition to that of p53 did not show further inhibition on MLN4924induced expression of cleaved caspase 3 compared with p53 knockdown alone (Supplementary Figure S11), implying that the impacts of p62 on MLN4924-induced apoptosis of hSMCs were completely dependent on p53. However, chromatin immunoprecipitation (ChIP) assays did not show any interaction between p53 protein and p62 promoter (Supplementary Figure S12), suggesting that p62 was not a target gene of p53.

p53 deficiency in SMCs blocks the inhibitory effects of MLN4924 on neointimal hyperplasia in mice. Finally, we addressed the question whether p53 in SMCs mediated the effects of MLN4924 in vivo. We established an inducible smooth muscle p53 knockout (SM-p53KO) mouse model. QRT-PCR results demonstrated significant decrease of p53 gene expression in aortas of these mice compared with littermate control (LC) mice after injection of tamoxifen (Figure 6a). LC and SM-p53KO mice were then subjected to femoral artery wire injury and treatment with MLN4924 or vehicle control. MLN4924 reduced intima/media ratio of injured femoral arteries compared with vehicle control in LC mice (Figures $6 \mathrm{~b}$ and $\mathrm{c}$ ). However, this reduction was blocked in SM-p53KO mice and as a result the intima/media ratio of MLN4924-treated SM-p53KO mice was significantly higher than that of MLN4924-treated LC mice (Figures $6 b$ and $c$ ). Consistently, the inhibitory impact of MLN4924 on SMApositive cells in the injured arteries was blocked by SMp53KO (Figures $6 \mathrm{~d}$ and e). TUNEL-positive cells were 
a

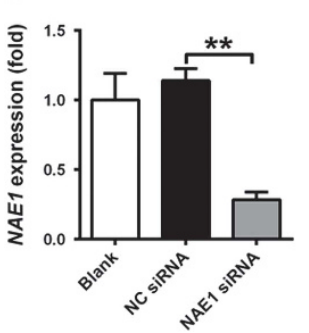

b

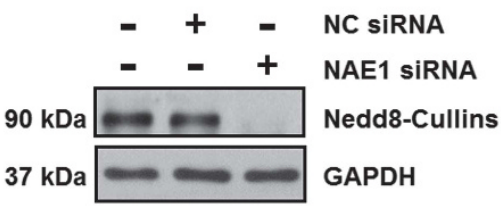

d

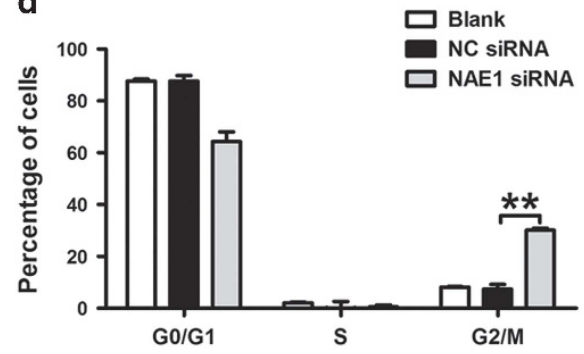

C
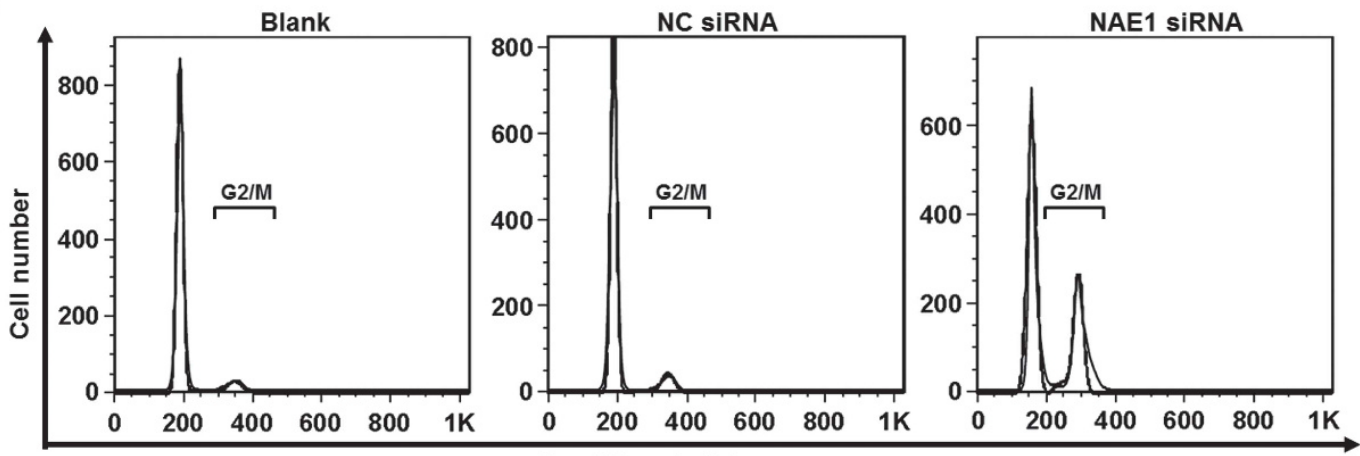

Propidium lodide

e
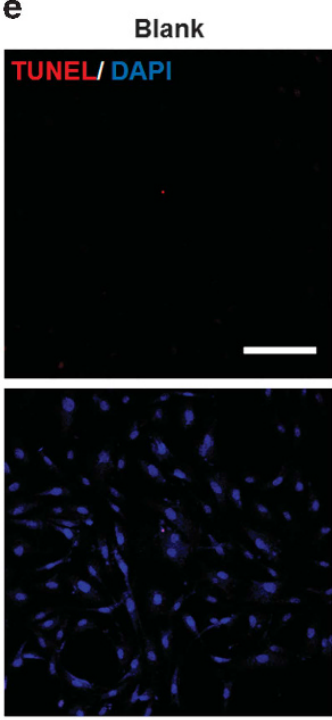

g

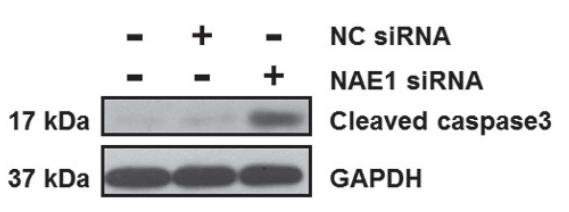

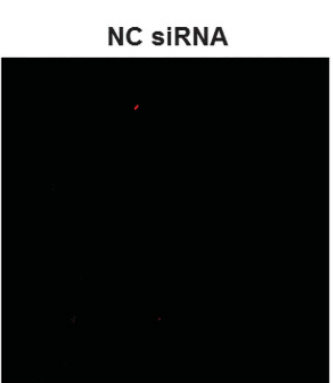
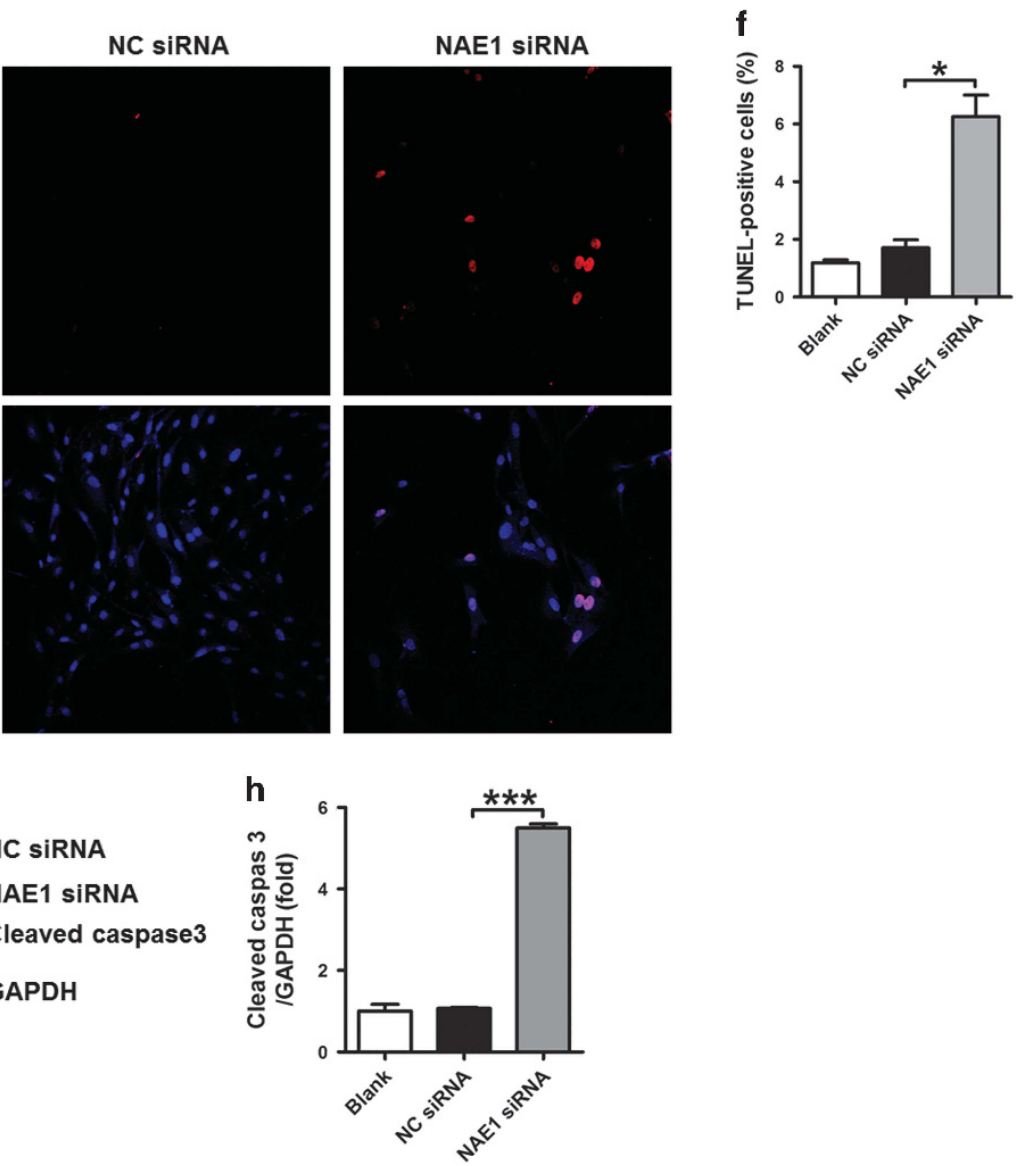

Figure 3 Knockdown of NAE1 induces G2/M arrest and apoptosis in hSMCs. (a) QRT-PCR verification of gene-specific silencing of NAE1 in hSMCs. NC siRNA: negative control siRNA. (b) Western blotting analysis of Nedd8-cullins in hSMCs. GAPDH was used as a loading control. (c) FACS analysis of cell cycle of hSMCs. (d) Quantification of the percentages of hSMCs in different cell cycle phases. (e) TUNEL staining of hSMCs. (f) Quantification of TUNEL-positive cells. (g) Western blotting analysis of cleaved caspase 3 in hSMCs. (h) Quantification of (g). Cells were transfected with siRNA for $12 \mathrm{~h}(\mathbf{a})$ or $24 \mathrm{~h}(\mathbf{b}-\mathbf{h})$, and then cultured for another $72 \mathrm{~h}$. Scale bar: $200 \mu \mathrm{m}$. Each experiment was repeated at least three times. ${ }^{*} P<0.05,{ }^{* *} P<0.01,{ }^{* *} P<0.001$ 


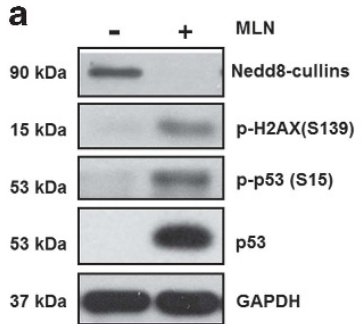

b

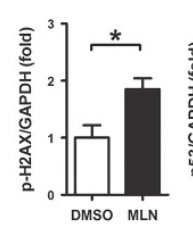

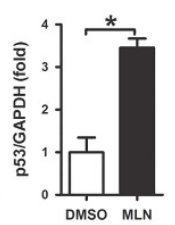

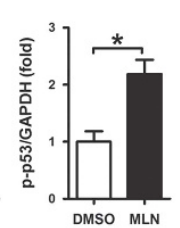

c

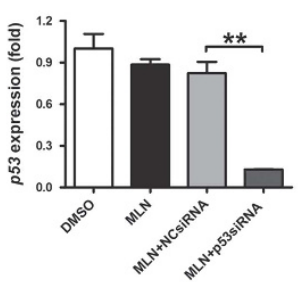

d

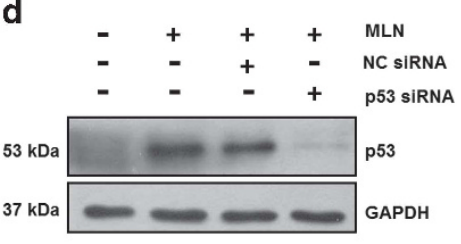

e

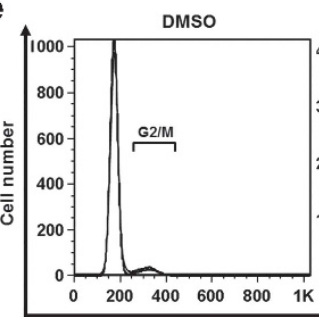

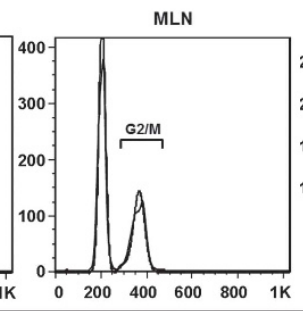
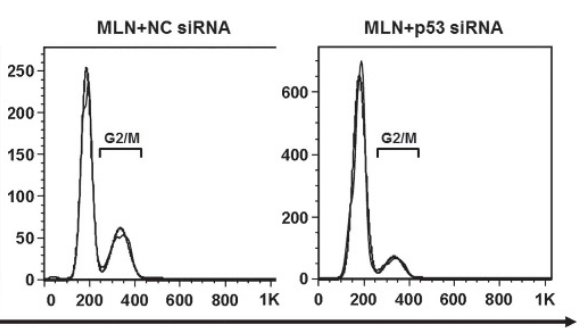

f

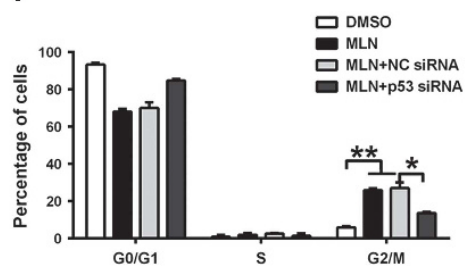

g
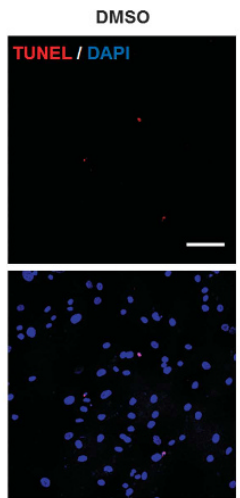

MLN

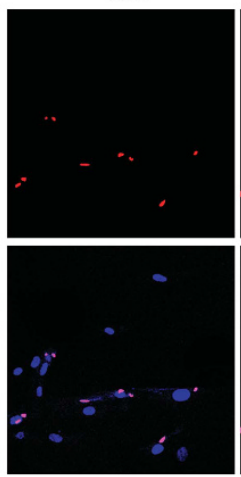

$M L N+N C$ siRNA

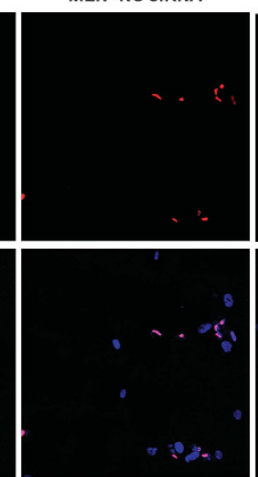

MLN+p53 siRNA

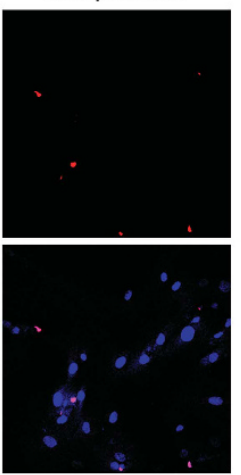

h

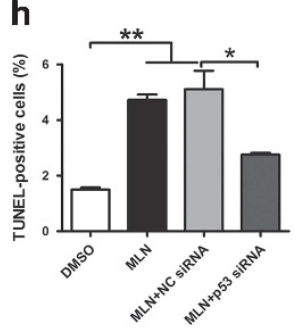

i

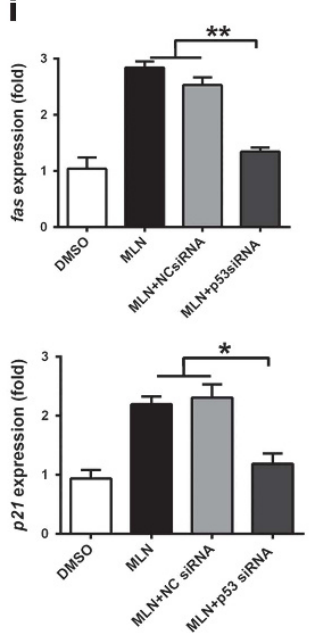

Figure 4 p53 mediates the effects of MLN4924 on G2/M arrest and apoptosis in hSMCs. (a) Western blotting analysis of Nedd8-cullins, p-H2AX, p-p53, and total p53 in hSMCs treated with vehicle (DMSO) or MLN4924 (MLN). GAPDH was used as a loading control. (b) Quantification of (a). (c) QRT-PCR verification of gene-specific silencing of p53 in hSMCs. NC siRNA: negative control siRNA. (d) Western blotting verification of gene-specific silencing of p53. (e) FACS analysis of cell cycle of hSMCs. (f) Quantification of percentages of hSMCs at different cell cycle phases. (g) TUNEL staining of hSMCs. (h) Quantification of TUNEL-positive cells. (i) QRT-PCR analysis of fas and p21. Cells were transfected with siRNA for $24 \mathrm{~h}$ and then treated for $72 \mathrm{~h}$. Scale bar: $300 \mu \mathrm{m}$. Each experiment was repeated at least three times. ${ }^{*} P<0.05,{ }^{* \star} P<0.01$

significantly increased by MLN4924 and this was also blocked by SM-p53KO (Figures $6 f$ and $g$ ). These results together suggested that MLN4924 inhibited neointimal hyperplasia and promoted apoptosis of VSMCs through p53 in vivo.

\section{Discussion}

Clinical practice demands novel strategies to further reduce the incidence of restenosis and therefore to achieve better outcomes after $\mathrm{PCl}$. Here in this study, we showed that inhibition of protein neddylation by MLN4924 was able to suppress neointimal hyperplasia in a mouse model of vascular injury. At the cellular and molecular level, inhibition of neddylation induced G2/M arrest and apoptosis of VSMCs depending on p53 and/or p62, reflecting the effects of MLN4924 in vivo.
Our data demonstrated neddylation as a potential novel therapeutic target for restenosis after $\mathrm{PCl}$. Drug-eluting stents that effectively suppress proliferation of VSMCs have markedly decreased the rate of stenosis. ${ }^{33,34}$ However, residual risk remains and new strategies utilizing new mechanisms are warranted. Targeting apoptosis of VSMCs represents an attractive approach. In the process of restenosis, proliferation and apoptosis are two parallel events that determine the net accumulation of VSMCs in the neointima. ${ }^{10}$ We revealed that inhibition of neddylation by MLN4924 effectively suppressed neointimal hyperplasia most likely through promoting apoptosis of VSMCs in a classical mouse model of vascular injury. These observations in mice were further elaborated by the in vitro data showing that inhibition of neddylation caused G2/ $\mathrm{M}$ arrest and apoptosis of VSMCs. Therefore, targeting apoptosis of VSMCs through inhibition of neddylation may 


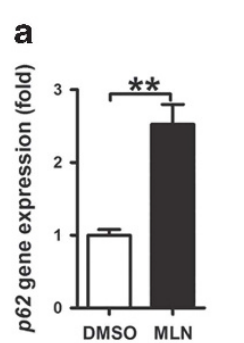

b

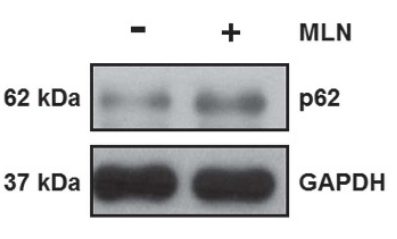

e
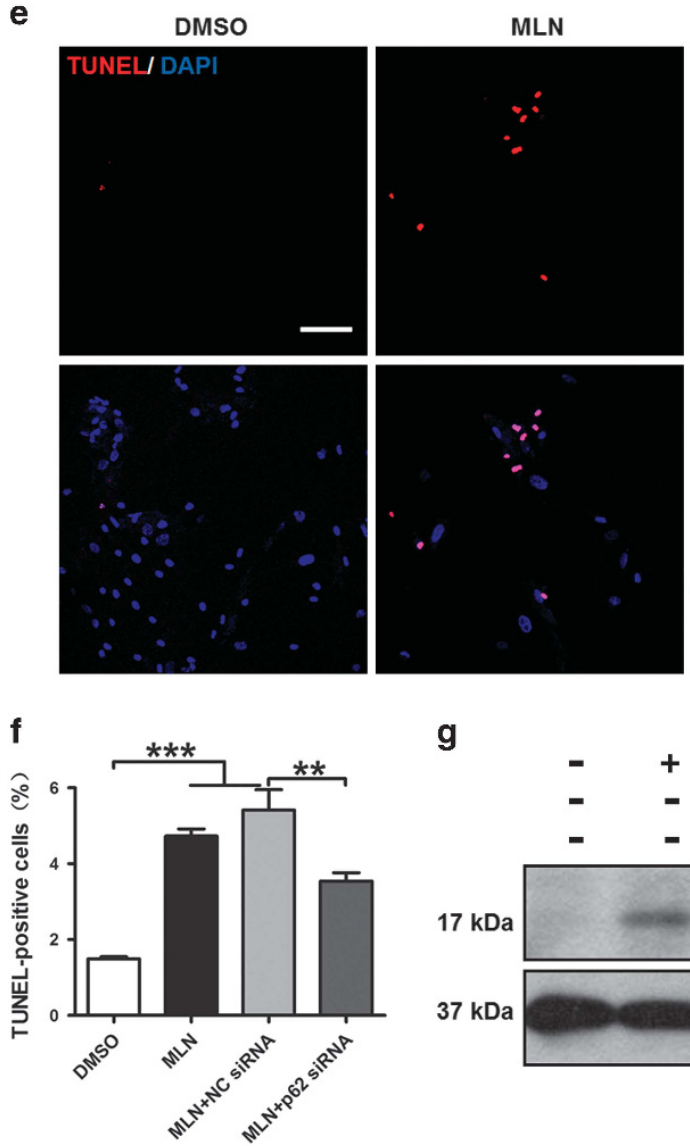

g
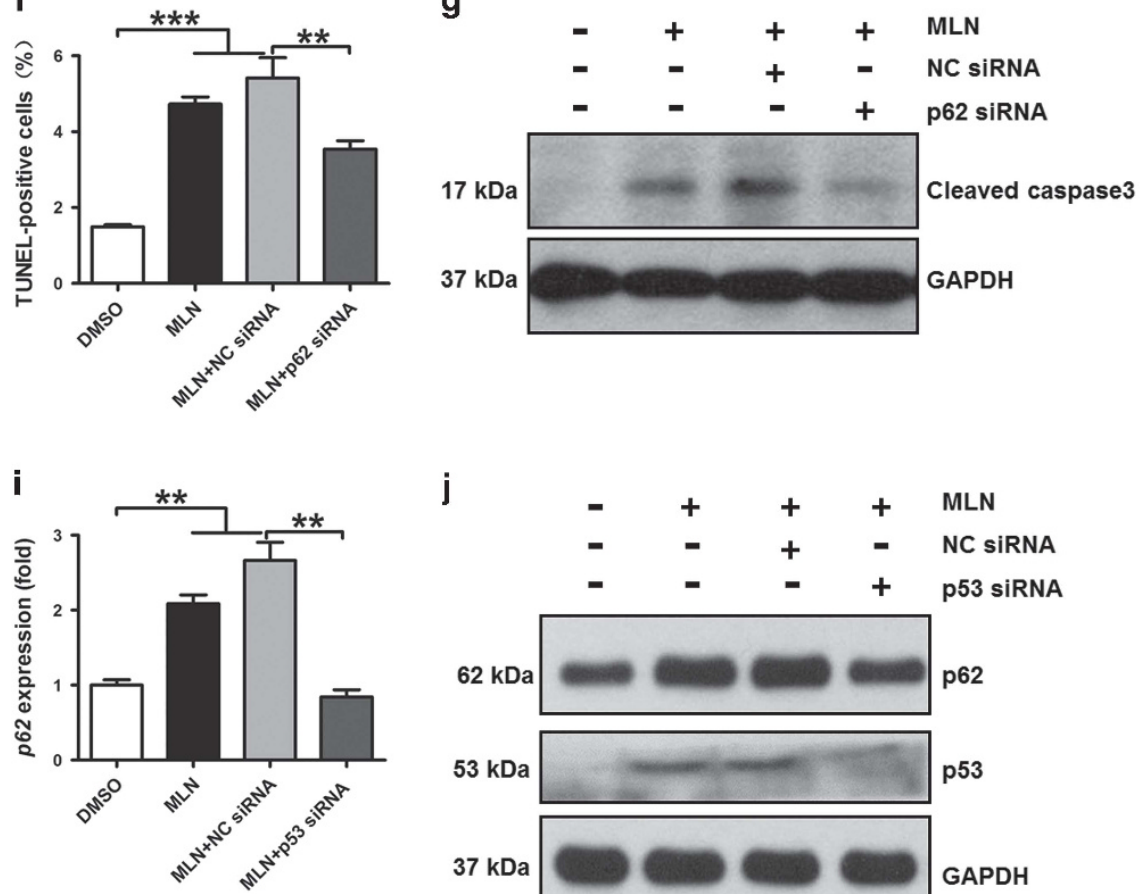

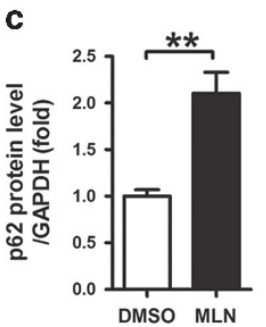

d

$62 \mathrm{kDa}$

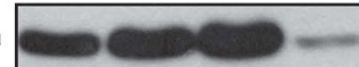

- NC siRNA

37 kDa

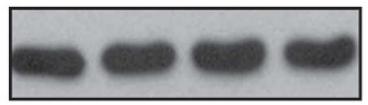

GAPDH
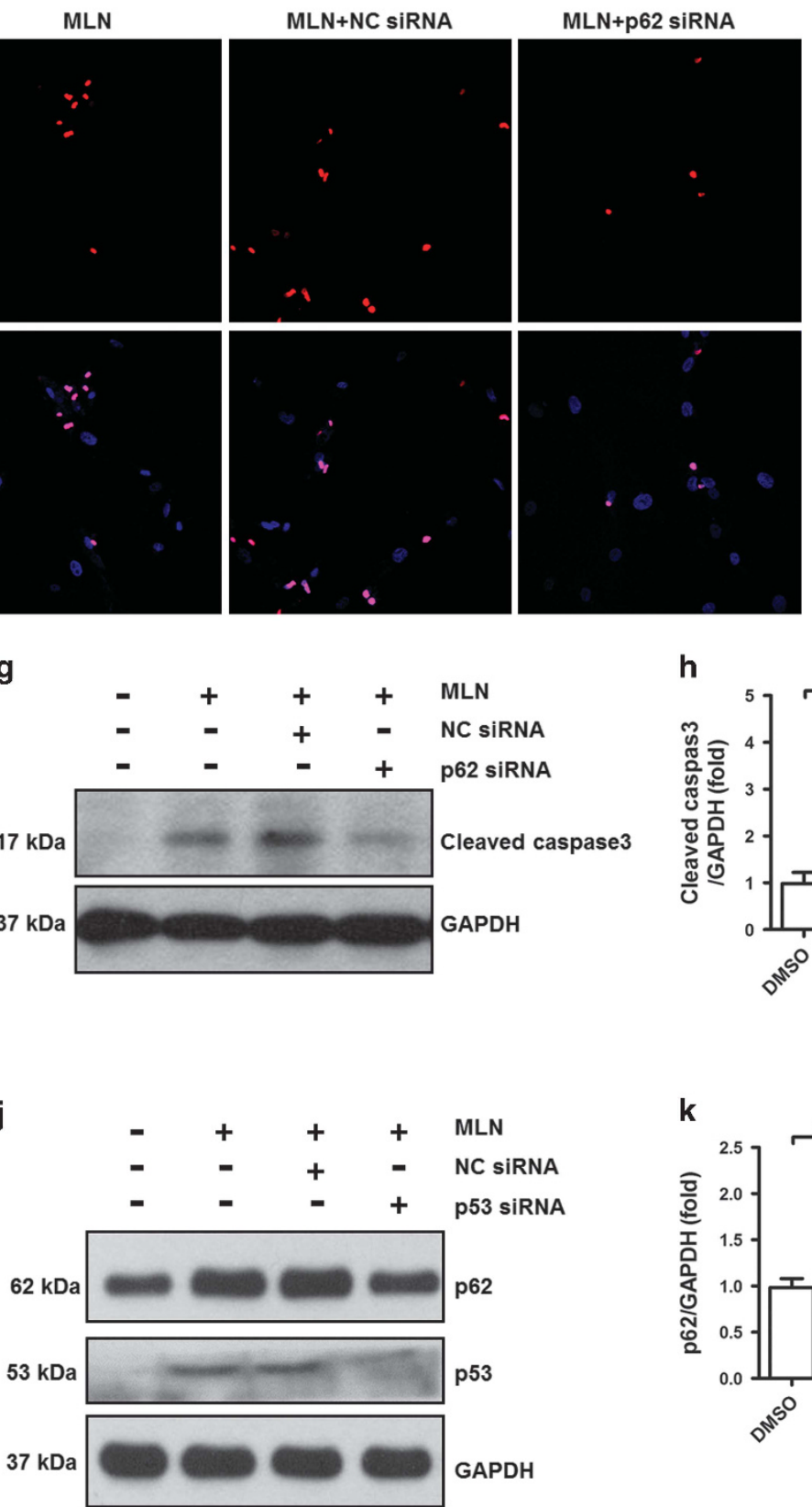

h

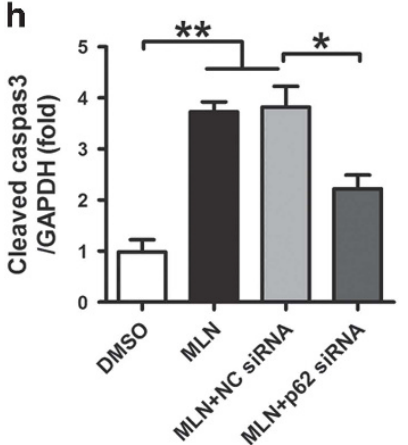

k

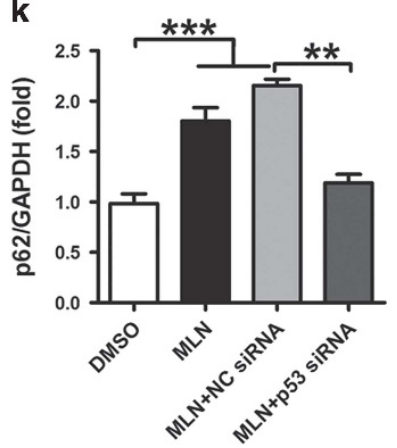

Figure 5 p62 mediates the pro-apoptotic effects of MLN4924 in hSMCs. (a) QRT-PCR analysis of gene expression of p62 in hSMCs treated with vehicle (DMSO) or MLN4924 (MLN). (b) Western blotting analysis of p62 in hSMCs treated with DMSO or MLN. GAPDH was used as a loading control. (c) Quantification of (b). (d) Western blotting verification of gene-specific silencing of p62 in hSMCs. NC siRNA: negative control siRNA. (e) TUNEL staining of hSMCs. (f) Quantification of TUNEL-positive cells. (g) Western blotting analysis of cleaved caspase 3 in hSMCs. (h) Quantification of (g). (i) QRT-PCR analysis of gene expression of p62 in hSMCs treated with DMSO or MLN and with NC siRNA or p53 siRNA. (j) Western blotting analysis of p62 and p53. (k) Quantification of p62 western blotting result. For (a-c), cells were treated for $72 \mathrm{~h}$. For other panels, cells were transfected with siRNA for $12 \mathrm{~h}(\mathbf{d}-\mathbf{h})$ or $24 \mathrm{~h}(\mathbf{i}-\mathbf{k})$, and then treated for $72 \mathrm{~h}$. Scale bar: $300 \mu \mathrm{m}$. Each experiment was repeated at least three times. ${ }^{*} P<0.05$, ${ }^{* *} P<0.01$, ${ }^{* * *} P<0.001$ 
326
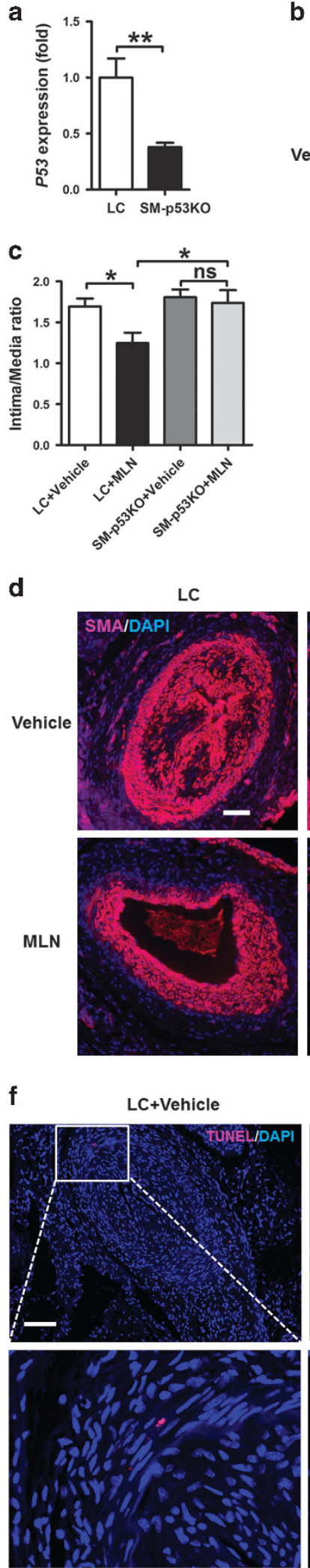

b

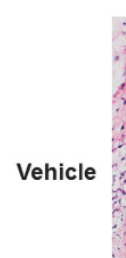

MLN
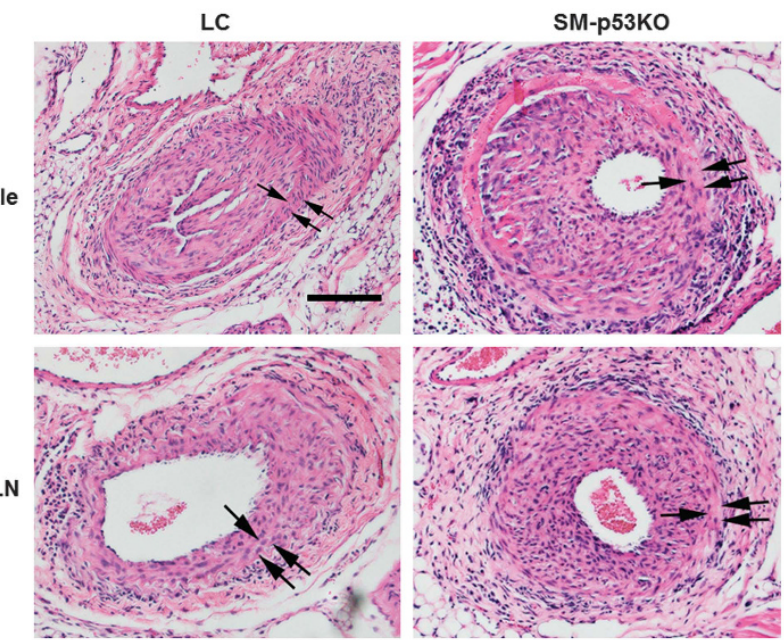

SM-р53ко
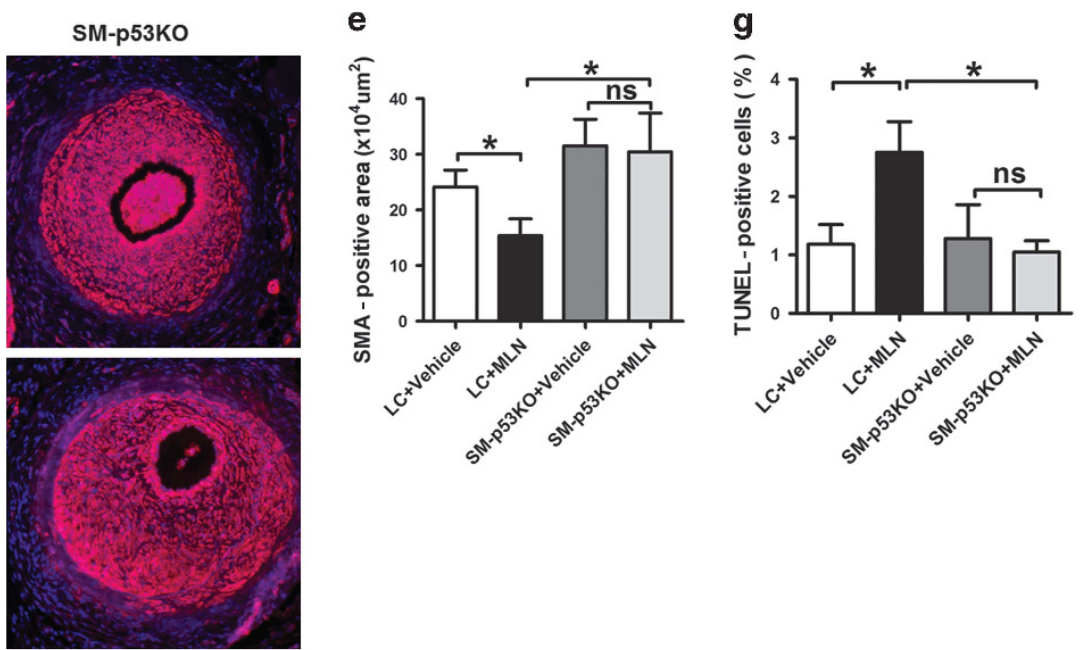

SM-p53KO+Vehicle
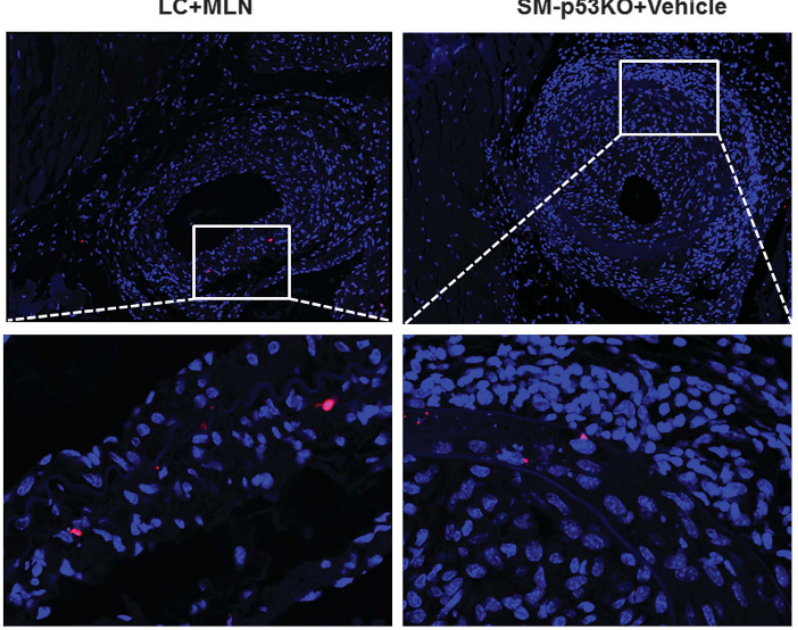

SM-p53KO+MLN
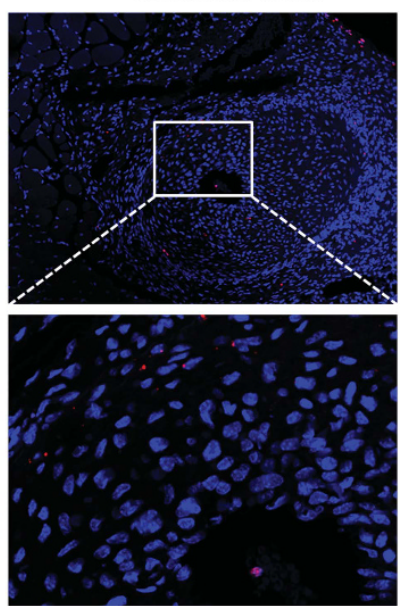
Figure 6 Smooth muscle p53 deficiency blocks the inhibitory effects of MLN4924 on neointimal hyperplasia in mice. (a) QRT-PCR analysis of p53 gene expression in aortas of littermate control (LC) mice and inducible smooth muscle p53 knockout (SM-p53KO) mice after tamoxifen injection. (b) Representative hematoxylin and eosin staining of crosssections of femoral arteries from wire-injured LC and SM-p53KO mice that were treated with vehicle or MLN4924 (MLN) for 28 days. Single arrows indicate internal elastic lamina and double arrows indicate external elastic lamina. (c) Quantification of intima/media ratio. (d) Representative immunofluorescence staining of SMA in injured arteries. (e) Quantification of SMA-positive cells. (f) Representative TUNEL staining of injured arteries. (g) Quantification of TUNEL index as percentage of apoptotic cells. Scale bars: $200 \mu$ m. $n=4$. ns, not significant.. $P<0.05,{ }^{* \star} P<0.01$

be a feasible strategy to further combat restenosis. It is worth to point out that the mechanism perturbing neddylation has been tested for cancer treatment and MLN4924 is already in clinical trials, ${ }^{35}$ which may expedite the application of the same mechanism in treating restenosis.

Mechanistically, p53 mediated the effects of MLN4924 on G2/M arrest and apoptosis of VSMCs as well as on neointimal hyperplasia in mice. We found that MLN4924 caused upregulation of both total and phosphorylation level of p53, as well as its transcriptional activity in VSMCs, likely through induction of DNA damage response. However, the mRNA level of p53 was not affected, suggesting that the regulation on p53 was at post-transcriptional level. Previous studies have demonstrated that DNA damage activates p53 by modifications including phosphorylation, ${ }^{36}$ leading to its accumulation and increase of transcriptional activation of pro-apoptotic factors such as fas, Puma, and Bax, ${ }^{37}$ as well as cell cycle regulation-related proteins such as $\mathrm{p} 21^{38,39}$ Our results unveiled similar mechanisms in VSMCs. Overexpression of p53 reduces and conversely deficiency of p53 aggravates neointima formation in mice. ${ }^{40-42}$ Our study further demonstrated that MLN4924 improved neointimal hyperplasia depending on p53-mediated apoptosis in SMCs. It has been well documented that p53 is critical player in both $\mathrm{G} 1 / \mathrm{S}$ arrest and G2/M arrest. ${ }^{43}$ However, in VSMCs upregulation of p53 has been reported to induce G2/M arrest in 2 days while induce G1/S arrest only after a much longer time (5 days). ${ }^{44}$ Similarly, our results demonstrated that upregulation of p53 by MLN4924 mainly caused G2/M arrest in VSMCs within 3 days.

There are both similarities and difference between the mechanisms of MLN4924 in VSMCs and cancer cells. Previous mechanistic studies have revealed that MLN4924 suppresses the growth of cancer cells through inactivation of Cullin-RING ligase (CRL) and subsequently inducing accumulation of $\mathrm{CRL}$ substrates such as Cdt1 and origin recognition complex subunit 1, both of which trigger DNA replication stress and induce DNA damage response. ${ }^{45,46}$ In addition, accumulation of other $\mathrm{CRL}$ substrates, including p21, ${ }^{45}$ NOXA,${ }^{47}$ and activating transcription factor $4,{ }^{48}$ has been identified to mediate cell cycle arrest and/or apoptosis and to inhibit the growth of cancer cells. Similarly, our data suggested that MLN4924 induced DNA damage response, cell cycle arrest, and apoptosis in VSMCs. However, a key difference exists between the mechanisms in VSMCs vs. cancer cells. In cancer cells, p53 deficiency does not inhibit MLN4924-induced apoptosis; instead, p53-deficient cancer cells are more susceptible to the drug. ${ }^{46}$ Our study revealed that p53 deficiency attenuated MLN4924-induced apoptosis and G2/M arrest in VSMCs, as well as blocked MLN4924induced suppression of neointimal hyperplasia in mice.

This study also found that p62 mediated the effects of MLN4924 on apoptosis of VSMCs in vitro. MLN4924 increased the expression of p62, which mediated the effects of MLN4924 on apoptosis. Previous studies have demonstrated that deficiency of p62 aggravates neointima formation in mice, indicating an important role of p62 in neointimal hyperplasia. ${ }^{49}$ Results in cancer cells have shown that MLN4924 causes accumulation of DEP domain containing MTOR interacting protein and hypoxia inducible factor 1 alpha that are in turn responsible for MLN4924-induced autophagy via inhibiting mTORC1. ${ }^{29}$ Our study did not find MLN4924 induce autophagy in VSMCs. Instead, MLN4924 increased the level of p62 mRNA likely through transcriptional regulation. Similar regulation of p62 at transcriptional level has been reported before. ${ }^{50}$ Interestingly, we demonstrated that the induction of p62 by MLN4924 was dependent on p53 in VSMCs, a novel mechanism that also mediated MLN4924-induced apoptosis. However, it remains to be further interrogated how p53 controls p62 expression in MLN4924-treated VSMCs and whether MLN4924 improves neointimal hyperplasia depending on p62 in vivo.

In conclusion, we have demonstrated that inhibition of neddylation induces VSMC apoptosis through p53/p62 and improves neointimal hyperplasia after mechanical vascular injury through p53 in mice. These findings support that regulation of neddylation is an attractive therapeutic approach to treat restenosis. Translational indications of these preclinical evidence support that MLN4924, an inhibitor of neddylation that has been tested for cancer therapy, is a strong candidate for novel treatments to further decrease the rate of restenosis after $\mathrm{PCl}$.

\section{Materials and Methods}

Animals and treatments. Eight weeks old male C57BL/6 mice were purchased from Shanghai SLAC Laboratory Animal Co. Ltd (Shanghai, China). Floxed p53 mice ${ }^{51}$ were crossed with SM-CreER ${ }^{T 2}$ mice $^{52}$ to generate p53 $3^{\mathrm{t} / \mathrm{fl} \text {; }}$ SM$\mathrm{CreER}^{\top 2}$-positive mice and LC mice (p53 $3^{\mathrm{flfl} / \mathrm{l}} ; \mathrm{SM}^{-C r e E R^{T 2}}$-negative). At 5 weeks old, p53 $3^{\mathrm{tl} / \mathrm{fl}}$; SM-CreER ${ }^{\mathrm{T}}$-positive mice were intraperitoneally injected with $1 \mathrm{mg}$ tamoxifen (Sigma-Aldrich, St. Louis, MO, USA) for 5 consecutive days to produce smooth muscle p53 knockout (SM-p53KO) mice. LC mice were injected with tamoxifen using the same protocol.

MLN4924 (30 mg/kg) or vehicle (2-hydroxypropyl-b-cyclodextrin, HPBCD) (HK388, Sangon Biotech, Shanghai, China) was administrated to 8 weeks old male mice via subcutaneous injection twice a day for 4 weeks after surgeries. All animal experiments were approved by the Institutional Review and Ethics Board of Ninth People's Hospital, Shanghai Jiao Tong University School of Medicine (HKDL 2016303) and the Institutional Animal Care and Use Committee of Institute for Nutritional Sciences, Shanghai Institutes for Biological Sciences, Chinese Academy of Sciences (2015-AN-10).

Femoral artery wire injury model. Femoral artery wire injury was performed according to methods previously described. ${ }^{53}$ In brief, mice were anesthetized with isoflurane and a $0.38 \mathrm{~mm}$ flexible angioplasty guide wire (Cook Inc, Bloomington, IN, USA) was inserted rotationally into the arterial lumen of right femoral arteries and then pulled back for a total of three times. After the third insertion the guide wire was left in place for $2 \mathrm{~min}$. Sham surgeries were performed on the left femoral arteries of the same mice. After 4 weeks, femoral arteries were 
harvested, fixed with 4\% paraformaldehyde, and embedded in paraffin. Serial crosssections $(5 \mu \mathrm{m})$ were cut for further analysis.

Histology and morphometric analysis. Cross-sections of femoral arteries were stained with hematoxylin and eosin. Intimal area and medial area were measured using Image $\mathrm{J}$ software (National Institutes of Health, Bethesda, MD, USA). The intima to media ratio was calculated.

Cell culture. hSMCs were obtained from the American Type Culture Collection (ATCC, Manassas, VA, USA). The cells were maintained in DMEM-F12 (Thermo Scientific, Grand Island, NY, USA) supplemented with 10\% FBS (Thermo Scientific), $100 \mathrm{U} / \mathrm{ml}$ penicillin, and $100 \mu \mathrm{g} / \mathrm{ml}$ streptomycin. hSMCs were treated with $1 \mu \mathrm{M}$ MLN4924 or DMSO for indicated length of time before various analysis.

Primary VSMCs were isolated from aortas of 8-week-old SM-p53KO and LC mice by enzyme digestion method. ${ }^{54}$ In brief, both thoracic and abdominal aortas were dissected from mice. Adventitia and endothelium were both removed. The samples were then cut into small pieces and digested with collagenase and elastase for $1 \mathrm{~h}$. The digestion was stopped with cultured media and cells were collected and cultured in DMEM-F12 supplemented with 20\% FBS, $100 \mathrm{U} / \mathrm{ml}$ penicillin, and $100 \mu \mathrm{g} / \mathrm{ml}$ streptomycin.

293FT cells (ATCC) were cultured with DMEM (Thermo Scientific) containing 10\% FBS, $100 \mathrm{U} / \mathrm{ml}$ penicillin, and $100 \mu \mathrm{g} / \mathrm{ml}$ streptomycin.

Immunofluorescence staining. Paraffin sections were deparaffinized, rehydrated, and subject to antigen retrieval. The sections were then blocked in $5 \%$ normal goat serum in PBS/Triton ( $0.3 \%$ Triton X-100 in PBS) at room temperature for $60 \mathrm{~min}$ and incubated with primary antibodies at $4{ }^{\circ} \mathrm{C}$ overnight. On the next day, the samples were incubated with fluorochrome-conjugated secondary antibodies (1:500, Thermo Scientific) for $1 \mathrm{~h}$ at $37^{\circ} \mathrm{C}$. Nuclei were stained by DAPI contained in mounting media (Thermo Scientific). hSMCs were seeded on coverslips and fixed with $4 \%$ paraformaldehyde at room temperature for $15 \mathrm{~min}$. The samples were then blocked in serum and incubated with primary antibodies and secondary antibodies sequentially. Fluorescent signals were captured using a confocal microscope. Primary antibodies for immunofluorescence were used at the following dilutions: anti- $\alpha$-SMA (Abcam, Cambridge, MA, USA) and anti-Ki67 (Cell Signaling Technology, Danvers, MA, USA).

TUNEL assay. For paraffin sections, TUNEL staining was done according to the manufacturer's protocol of In Situ Cell Death Detection Kit-TMR red (12156792910, Roche Diagnostics, Indianapolis, IN, USA). For cells, hSMCs were seeded on coverslips that were further used for TUNEL staining according to the manufacturer's protocol. Fluorescent signals were captured using a confocal microscope.

PI staining and FACS analysis. hSMCs were harvested using $0.25 \%$ trypsin, centrifuged, washed in PBS, and then fixed in $70 \%$ ethanol at $-80^{\circ} \mathrm{C}$ overnight. The cells were subsequently stained with propidium iodide (PI) (P4864, Sigma-Aldrich) solution containing $0.05 \mathrm{mg} / \mathrm{ml} \mathrm{PI}, 0.1 \%$ Triton $\mathrm{x}-100$, and $0.1 \mathrm{mg} / \mathrm{ml}$ ribonuclease $\mathrm{A}$ in PBS for $15 \mathrm{~min}$ at room temperature in dark. Cell cycle distribution was determined by Quanta SC (Beckman, Fullerton, CA, USA). Data were analyzed with Flow Jo software.

Quantitative RT-PCR. Total RNA from cells was extracted using TRIZOL (Thermo Scientific) and cDNA synthesis was accomplished using reverse transcription kits (Takara, Shiga, Japan) according to the manufacturer's protocol. Quantitative PCR was performed using SYBR Green Mix (Thermo Scientific) on an ABI7900HI (Applied Biosystems, Bartlesville, Oklahoma). Relative expression was determined via the Ct method and normalized to GAPDH as an internal control. Primer sequences were listed in Supplementary Table 1.

Total protein extraction and western blotting analysis. Cells were lysed with lysis buffer containing protease inhibitors. After quantification protein samples were separated by 10 or $15 \%$ SDS-PAGE, transferred to PVDF membranes (Millipore, Darmstadt, Germany), and analyzed by immunoblotting. The western blotting results were quantified using Image $\mathrm{J}$ software.

Primary antibodies were used at the following dilutions: GAPDH $(1: 10000$, KangChen Bio-tech, Shanghai, China), Nedd8 (1:1000, Cell Signaling Technology), cyclin b1 (1:1000, Santa Cruz, Dallas, TX, USA), p-cdc2 (1:1000, Santa Cruz), cleaved caspase 3 (1:500, Cell Signaling Technology), $\alpha$-tubulin (1:1000, Sigma),
p-H2AX (1:1000, Millipore), p-p53 (1:1000, Proteintech, Rosemont, IL, USA), p53 (1:1000, BD Biosciences, San Jose, CA, USA), p62 (1:1000, Cell Signaling Technology), LC3 (1:1000, Cell Signaling Technology).

siRNA silencing. hSMCs were transfected with siRNA using Lipofectamine 2000 (Thermo Scientific, Carlsbad, CA, USA) according to the manufacturer's protocol. Cdt1 siRNA was purchased from Santa Cruz Biotechnology and transfected according to manufacturer's instructions. The other siRNAs were synthesized by GenePharma (Shanghai, China). The sequences of the siRNAs were:

NC siRNA (human): 5'-UUCUCCGAACGUGUCACGUTT- 3'

p62 siRNA (human): 5'-GCATTGAAGTTGATATCGAdTdT- 3'

p53 siRNA (human): 5'-CUACUUCCUGAAAACAACG-3'

NAE1 siRNA (human): 5'-GGGUUGUGCUUUAGUCUGU-3'

ChIP. ChIP experiments were performed in 293FT cells using EZ-ChIP kit (Millipore) according to the manufacturer's protocols. Primer sequences for PCR analysis of ChIP products were: 5'-GAAATGAGAGGCTGGTCCAT-3', 5'-TTCTCGCCTCTGACTCCTTC3'

Statistical analysis. All results were presented as mean \pm S.E.M. and the statistical significance of differences between groups was assessed using Prism software (Graph Pad, La Jolla, CA, USA). Pair-wise comparisons were analyzed using student's $t$-test. $P$-values of $<0.05$ were considered statistically significant.

\section{Conflict of Interest}

The authors declare no conflict of interest.

Acknowledgements. This work was supported by grants from the National Natural Science Foundation of China (31671181, 91339110, 31371153), Science and Technology Commission of Shanghai Municipality (15140904400), and the Shanghai Summit \& Plateau Discipline Developing Projects to SZD; the National Natural Science Foundation of China (91539107) to JL.

1. Scarsini R, Zivelonghi C, Pesarini G, Vassanelli C, Ribichini FL. Repeat revascularization: percutaneous coronary intervention after coronary artery bypass graft surgery. Cardiovasc Revasc Med 2016; 17: 272-278.

2. de Quadros AS, Lima TC, Rodrigues AP, Modkovski TB, Welter DI, Sarmento-Leite R et al. Quality of life and health status after percutaneous coronary intervention in stable angina patients: results from the real-world practice. Catheter Cardiovasc Interv 2011; 77: 954-960.

3. Jukema JW, Verschuren JJ, Ahmed TA, Quax PH. Restenosis after PCl. Part 1: pathophysiology and risk factors. Nat Rev Cardiol 2012; 9: 53-62.

4. Marx SO, Totary-Jain H, Marks AR. Vascular smooth muscle cell proliferation in restenosis. Circ Cardiovasc Interv 2011; 4: 104-111.

5. Chaabane C, Otsuka F, Virmani R, Bochaton-Piallat ML. Biological responses in stented arteries. Cardiovasc Res 2013; 99: 353-363.

6. Sousa JE, Serruys PW, Costa MA. New frontiers in cardiology: drug-eluting stents: Part I. Circulation 2003; 107: 2274-2279.

7. Sousa JE, Serruys PW, Costa MA. New frontiers in cardiology: drug-eluting stents: Part II. Circulation 2003; 107: 2383-2389.

8. Cassese S, Byrne RA, Tada T, Pinieck S, Joner M, Ibrahim T et al. Incidence and predictors of restenosis after coronary stenting in 10004 patients with surveillance angiography. Heart 2014; 100: 153-159.

9. Alfonso F, Nuccio J, Cuevas C, Cardenas A, Gonzalo N, Jimenez-Quevedo P. Treatment of coronary in-stent restenosis with bioabsorbable vascular scaffolds. J Am Coll Cardiol 2014; 63: 2875.

10. Bennett MR. Apoptosis of vascular smooth muscle cells in vascular remodelling and atherosclerotic plaque rupture. Cardiovasc Res 1999; 41: 361-368.

11. Sata M, Perlman H, Muruve DA, Silver M, Ikebe M, Libermann TA et al. Fas ligand gene transfer to the vessel wall inhibits neointima formation and overrides the adenovirusmediated T cell response. Proc Natl Acad Sci USA 1998; 95: 1213-1217.

12. Katsiki N, Tziomalos K, Chatzizisis $\mathrm{Y}$, Elisaf M, Hatzitolios Al. Effect of HMG-CoA reductase inhibitors on vascular cell apoptosis: beneficial or detrimental? Atherosclerosis 2010; 211: 9-14.

13. Deuse $T$, Hua $X$, Wang D, Maegdefessel L, Heeren J, Scheja $L$ et al. Dichloroacetate prevents restenosis in preclinical animal models of vessel injury. Nature 2014; 509: 641-644.

14. Enchev RI, Schulman BA, Peter M. Protein neddylation: beyond cullin-RING ligases. Nat Rev Mol Cell Biol 2015; 16: 30-44.

15. Kandala S, Kim IM, Su H. Neddylation and deneddylation in cardiac biology. Am J Cardiovasc Dis 2014; 4: 140-158.

16. Rabut G, Peter M. Function and regulation of protein neddylation. 'Protein modifications: beyond the usual suspects' review series. EMBO Rep 2008; 9: 969-976. 
17. Watson IR, Irwin MS, Ohh M. NEDD8 pathways in cancer, Sine Quibus Non. Cancer Cell 2011; 19: 168-176.

18. Brownell JE, Sintchak MD, Gavin JM, Liao H, Bruzzese FJ, Bump NJ et al. Substrateassisted inhibition of ubiquitin-like protein-activating enzymes: the NEDD8 E1 inhibitor MLN4924 forms a NEDD8-AMP mimetic in situ. Mol Cell 2010; 37: 102-111.

19. Soucy TA, Smith PG, Milhollen MA, Berger AJ, Gavin JM, Adhikari S et al. An inhibitor of NEDD8-activating enzyme as a new approach to treat cancer. Nature 2009; 458: 732-767.

20. Luo Z, Yu G, Lee HW, Li L, Wang L, Yang D et al. The Nedd8-activating enzyme inhibitor MLN4924 induces autophagy and apoptosis to suppress liver cancer cell growth. Cancer Res 2012; 72: 3360-3371.

21. Lin JJ, Milhollen MA, Smith PG, Narayanan U, Dutta A. NEDD8-targeting drug MLN4924 elicits DNA rereplication by stabilizing Cdt1 in S phase, triggering checkpoint activation, apoptosis, and senescence in cancer cells. Cancer Res 2010; 70: 10310-10320.

22. Gu Y, Kaufman JL, Bernal L, Torre C, Matulis SM, Harvey RD et al. MLN4924, an NAE inhibitor, suppresses AKT and mTOR signaling via upregulation of REDD1 in human myeloma cells. Blood 2014; 123: 3269-3276.

23. Halayko AJ, Rector E, Stephens NL. Airway smooth muscle cell proliferation: characterization of subpopulations by sensitivity to heparin inhibition. Am J Physiol 1998; 274: L17-L25.

24. Booher RN, Alfa CE, Hyams JS, Beach DH. The fission yeast cdc2/cdc13/suc1 protein kinase: regulation of catalytic activity and nuclear localization. Cell 1989; 58: 485-497.

25. Paiva C, Godbersen JC, Berger A, Brown JR, Danilov AV. Targeting neddylation induces DNA damage and checkpoint activation and sensitizes chronic lymphocytic leukemia B cells to alkylating agents. Cell Death Dis 2015; 6: e1807.

26. Blow JJ, Dutta A. Preventing re-replication of chromosomal DNA. Nat Rev Mol Cell Biol 2005; 6: $476-486$.

27. Fujita M. Cdt1 revisited: complex and tight regulation during the cell cycle and consequences of deregulation in mammalian cells. Cell Div 2006; 1: 22.

28. Vousden KH, Lu X. Live or let die: the cell's response to p53. Nat Rev Cancer 2002; 2 594-604

29. Zhao $Y$, Xiong $X$, Jia L, Sun $Y$. Targeting Cullin-RING ligases by MLN4924 induces autophagy via modulating the HIF1-REDD1-TSC1-mTORC1-DEPTOR axis. Cell Death Dis 2012; 3: e386.

30. Klionsky DJ, Abeliovich H, Agostinis P, Agrawal DK, Aliev G, Askew DS et al. Guidelines for the use and interpretation of assays for monitoring autophagy in higher eukaryotes. Autophagy 2008; 4: 151-175.

31. Zhang YB, Gong JL, Xing TY, Zheng SP, Ding W. Autophagy protein p62/SQSTM1 is involved in HAMLET-induced cell death by modulating apotosis in U87MG cells. Cell Death Dis 2013; 4: e550.

32. Jin Z, Li Y, Pitti R, Lawrence D, Pham VC, Lill JR et al. Cullin3-based polyubiquitination and p62-dependent aggregation of caspase-8 mediate extrinsic apoptosis signaling. Cell 2009; 137: $721-735$.

33. Morice MC, Serruys PW, Sousa JE, Fajadet J, Ban Hayashi E, Perin M et al. A randomized comparison of a sirolimus-eluting stent with a standard stent for coronary revascularization. N Engl J Med 2002; 346: 1773-1780

34. Stone GW, Ellis SG, Cox DA, Hermiller J, O'Shaughnessy C, Mann JT et al. A polymerbased, paclitaxel-eluting stent in patients with coronary artery disease. N Engl J Med 2004 350: 221-231

35. Nawrocki ST, Griffin P, Kelly KR, Carew JS. MLN4924: a novel first-in-class inhibitor of NEDD8-activating enzyme for cancer therapy. Expert Opin Investig Drugs 2012; 21: 1563-1573.

36. Lakin ND, Jackson SP. Regulation of p53 in response to DNA damage. Oncogene 1999; 18 7644-7655.
37. Roos WP, Kaina B. DNA damage-induced cell death by apoptosis. Trends Mol Med 2006; 12 440-450.

38. He GG, Siddik ZH, Huang ZF, Wang RN, Koomen J, Kobayashi R et al. Induction of p21 by p53 following DNA damage inhibits both Cdk4 and Cdk2 activities. Oncogene 2005; 24 : 2929-2943.

39. Macleod KF, Sherry N, Hannon G, Beach D, Tokino T, Kinzler K et al. P53-dependent and independent expression of P21 during cell-growth, differentiation, and DNA-damage. Genes Dev 1995; 9: 935-944.

40. Wan S, George SJ, Nicklin SA, Yim APC, Baker AH. Overexpression of p53 increases lumen size and blocks neointima formation in porcine interposition vein grafts. Mol Ther 2004; 9 : 689-698.

41. George SJ, Angelini GD, Capogrossi MC, Baker AH. Wild-type p53 gene transfer inhibits neointima formation in human saphenous vein by modulation of smooth muscle cell migration and induction of apoptosis. Gene Ther 2001; 8: 668-676.

42. Sata M, Tanaka K, Ishizaka N, Hirata Y, Nagai R. Absence of $p 53$ leads to accelerated neointimal hyperplasia after vascular injury. Arterioscler Thromb Vasc Biol 2003; 23 : 1548-1552.

43. Agarwal ML, Agarwal A, Taylor WR, Stark GR. P53 controls both the $G(2) / M$ and the $G(1)$ cell-cycle checkpoints and mediates reversible growth arrest in human fibroblasts. Proc Nat Acad Sci USA 1995; 92: 8493-8497.

44. Yonemitsu Y, Kaneda Y, Tanaka S, Nakashima Y, Komori K, Sugimachi K et al. Transfer of wild-type p53 gene effectively inhibits vascular smooth muscle cell proliferation in vitro and in vivo. Circ Res 1998; 82: 147-156.

45. Lan $H$, Tang Z, Jin H, Sun Y. Neddylation inhibitor MLN4924 suppresses growth and migration of human gastric cancer cells. Sci Rep 2016; 6: 24218.

46. Lin JJ, Milhollen MA, Smith PG, Narayanan U, Dutta A, NEDD8-Targeting Drug MLN4924 elicits DNA rereplication by stabilizing Cdt1 in $S$ phase, triggering checkpoint activation, apoptosis, and senescence in cancer cells. Cancer Res 2010; 70 10310-10320

47. Yao WT, Wu JF, Yu GY, Wang R, Wang K, Li LH et al. Suppression of tumor angiogenesis by targeting the protein neddylation pathway. Cell Death Dis 2014; 5: e1059.

48. Chen P, Hu T, Liang Y, Li P, Chen X, Zhang J et al. Neddylation inhibition activates the extrinsic apoptosis pathway through ATF4-CHOP-DR5 axis in human esophageal cancer cells. Clin Cancer Res 2016; 22: 4145-4157.

49. Sugimoto R, Warabi E, Katayanagi S, Sakai S, Uwayama J, Yanagawa T et al. Enhanced neointimal hyperplasia and carotid artery remodelling in sequestosome 1 deficient mice. J Cell Mol Med 2010; 14: 1546-1554.

50. Jain A, Lamark T, Sjottem E, Larsen KB, Awuh JA, Overvatn A et al. p62/SQSTM1 is a target gene for transcription factor NRF2 and creates a positive feedback loop by inducing antioxidant response element-driven gene transcription. J Biol Chem 2010; 285: 22576-22591.

51. Marino S, Vooijs M, van Der Gulden H, Jonkers J, Berns A. Induction of medulloblastomas in p53-null mutant mice by somatic inactivation of $\mathrm{Rb}$ in the external granular layer cells of the cerebellum. Genes Dev 2000; 14: 994-1004.

52. Wendling O, Bornert JM, Chambon P, Metzger D. Efficient temporally-controlled targeted mutagenesis in smooth muscle cells of the adult mouse. Genesis 2009; 47: 14-18.

53. Sun JY, Li C, Shen ZX, Zhang WC, Ai TJ, Du LJ et al. Mineralocorticoid receptor deficiency in macrophages inhibits neointimal hyperplasia and suppresses macrophage inflammation through SGK1-AP1/NF-kappaB pathways. Arterioscler Thromb Vasc Biol 2016; 36 : 874-885.

54. Guo X, Shi N, Cui XB, Wang JN, Fukui Y, Chen SY. Dedicator of cytokinesis 2, a novel regulator for smooth muscle phenotypic modulation and vascular remodeling. Circ Res 2015; 116: E71-E80.

Supplementary Information accompanies this paper on Cell Death and Differentiation website (http://www.nature.com/cdd) 